\title{
Probabilistic Reasoning Across the Causal Hierarchy
}

\author{
Duligur Ibeling, ${ }^{1}$ Thomas Icard ${ }^{2}$ \\ ${ }^{1}$ Department of Computer Science, Stanford University \\ ${ }^{2}$ Department of Philosophy, Stanford University \\ \{duligur, icard\}@stanford.edu
}

\begin{abstract}
We propose a formalization of the three-tier causal hierarchy of association, intervention, and counterfactuals as a series of probabilistic logical languages. Our languages are of strictly increasing expressivity, the first capable of expressing quantitative probabilistic reasoning-including conditional independence and Bayesian inference-the second encoding docalculus reasoning for causal effects, and the third capturing a fully expressive $d o$-calculus for arbitrary counterfactual queries. We give a corresponding series of finitary axiomatizations complete over both structural causal models and probabilistic programs, and show that satisfiability and validity for each language are decidable in polynomial space.
\end{abstract}

\section{Introduction and Summary}

Intelligence commonly involves prediction, anticipating future events on the basis of past observations (e.g., "Will the water pipes freeze again this winter?"). Intelligent planning and decision-making additionally require predicting what would happen under a hypothetical action ("Will the pipes freeze if we keep the heat on?"). An even more sophisticated ability-critical for tasks like explanation-is to reason counterfactually about what would have happened given knowledge about what in fact happened ("Would the pipes have frozen if we had left the heat on, given that the heat was off and the pipes in fact froze?"). These three modes of reasoning constitute a causal hierarchy (Shpitser and Pearl 2008; Pearl 2009), highlighting the significance of structural causal knowledge for flexible thought and action.

The aim of the present article is to gain conceptual as well as technical insight into this hierarchy by employing tools from logic. Loosely following earlier work (Shpitser and Pearl 2008), we propose a characterization of its levels in terms of logical syntax. Semantically, all three languages are interpreted over the same class of models, namely structural causal models (and later probabilistic programs). The languages differ in how much they can express about these models. $\mathcal{L}_{1}$, the language of association, expresses only "pure" probabilistic facts and relationships; $\mathcal{L}_{2}$, the language of probabilistic intervention, allows expressing probabilities

Copyright (C) 2020, Association for the Advancement of Artificial Intelligence (www.aaai.org). All rights reserved. of basic conditional "if...then..." statements; $\mathcal{L}_{3}$, the language of probabilistic counterfactuals, encodes probabilities for arbitrary boolean combinations of such conditional statements. Using standard ideas from logic and existing results, we can address questions about definability and expressiveness. For instance, it is easy to prove in our framework that each language is strictly more expressive than those below it in the hierarchy (Prop. 1, 2 below). We can also interpret well-known insights from the graphical models and causal learning literatures as graph definability results for appropriate probabilistic logical languages, analogously to correspondence theory in modal logic (van Benthem 2001).

In possession of a precise syntax and semantics for probabilistic causal reasoning, questions of axiomatization naturally arise. That is, we would like to identify a perspicuous set of basic principles that underly all such reasoning. One of our main technical contributions is a series of finitary (sound and complete) axiomatizations for each level of the causal hierarchy (Thm. 5), relying on methods from semialgebraic geometry. As a corollary to these completeness results, we also reveal a "small-model property" with the consequence that satisfiability and validity for $\mathcal{L}_{1}, \mathcal{L}_{2}$, and $\mathcal{L}_{3}$ can be decided in polynomial space (Thm. 11).

Finally, in the last part of the paper we consider an alternative interpretation for our three logical languages. Probabilistic programs, with an appropriate notion of causal intervention, provide a procedural semantics for probabilistic counterfactual claims and queries. We establish an equivalence between these models and a natural subclass of computable structural causal models (Thm. 12). The equivalence in turn implies soundness and completeness of our axiomatizations with respect to this interpretation as well.

\section{Relation to Previous Work}

While deterministic causal counterfactuals and probabilistic logics have both received extensive treatment independently, the present contribution appears to be the first systematic study of probabilistic counterfactuals. Our work thus synthesizes and improves upon a long line of previous work. Axioms for causal conditionals interpreted over structural causal models are well understood (Galles and Pearl 1998; Halpern 2000; Pearl 2009; Zhang 2013; Ibeling and Icard 
2019) and play a distinct role in causal reasoning tasks such as identification (Shpitser and Pearl 2008; Pearl and Bareinboim 2012). Indeed, some prominent approaches to causal learning and identification even employ algorithmic logicbased techniques (Hyttinen, Eberhardt, and Järvisalo 2014; 2015; Triantafillou and Tsamardinos 2015).

Meanwhile, much is known about formalized probability calculi. (Fagin, Halpern, and Megiddo 1990) considered a probability logic built over a language of polynomials sufficiently expressive to encompass essentially all ordinary probabilistic reasoning about propositional facts, including Bayesian inference, conditional independence, and so on. However they left open the problem of explicit axiomatization. A complete axiomatization was later provided by (Perović et al. 2008) using an infinitary proof rule. Whereas our main interest is causal reasoning beyond the first level of the hierarchy, Thm. 5 incidentally establishes the first (weakly) complete finitary axiomatization for "pure" probability logic over a language of polynomials. Moving to the second and third levels of the hierarchy, Thm. 5 also presents the first combined axiomatization for probabilistic reasoning about causal counterfactuals.

(Fagin, Halpern, and Megiddo 1990) established a complexity upper-bound (PSPACE) for their satisfiability problem. On all three levels of the hierarchy, we obtain the same upper bound for our decision problem (Thm. 11). Both arguments rely crucially on the procedure given by (Canny 1988) to decide the existential theory of a real closed field. It has been previously suggested to apply cylindrical algebraic decomposition (which decides the full first-order theory of real closed fields) to causal questions (Geiger and Meek 1999).

Encoding causal knowledge in an implicit way via a generative probabilistic program has been explored recently by a number of research groups (Lake et al. 2017; Bingham et al. 2019; Tavares et al. 2019). Deterministic conditionals over "simulation programs" have been axiomatized (Ibeling and Icard 2018), showing that in general such an interpretation validates strictly fewer principles than structural causal models. This weaker axiomatic system was also embedded in a probability logic with linear inequalities (Ibeling 2018). It is possible, however, to restrict the class of probabilistic programs so as to ensure equivalence with (an appropriate class of) structural causal models (Ibeling and Icard 2019). We draw on all of this work in what follows.

\section{Causal Models}

We are interested in structural causal models (Spirtes, Glymour, and Scheines 2000; Pearl 2009) defined over a set $\mathcal{X}$ of endogenous variables and a set $\mathcal{U}$ of exogenous variables; let the combined variable set be $\mathcal{V}=\mathcal{X} \cup \mathcal{U}$. Every variable $V \in \mathcal{V}$ takes on a value from an admissible set $\operatorname{Val}(V)$. While $\mathcal{X}$ and $\mathcal{U}$ may be infinite, we assume $\operatorname{Val}(X)$ is finite for all $X \in \mathcal{X}$.

Definition 1 (Structural Causal Model). We define a SCM to be a pair $\mathfrak{M}=(\mathcal{F}, P)$, where $P$ is a probability measure on a $\sigma$-algebra $\Sigma$ of settings $\mathfrak{u}$ of $\mathcal{U}$, and $\mathcal{F}=\left\{f_{X}\right\}_{X \in \mathcal{X}}$ is a set of functions, one for each endogenous variable.

Given a set of variables $\mathcal{V}^{\prime} \subseteq \mathcal{V}$, we call an assignment of each variable $V \in \mathcal{V}^{\prime}$ to a value in $\operatorname{Val}(V)$ a setting. Thus every $f_{X}$ is a function from settings $\mathfrak{x}$ of endogenous variables $\mathcal{X}$ and settings $\mathfrak{u}$ of exogenous variables $\mathcal{U}$ to a value $f_{X}(\mathfrak{x}, \mathfrak{u}) \in \operatorname{Val}(X) . P(\mathcal{U})$ and $\mathcal{F}$ thus define the obvious joint probability distribution $P_{\mathfrak{M}}(\mathcal{X})$.

Definition 2 (Intervention). An intervention is a partial function $i: X \mapsto \operatorname{Val}(X)$. It specifies variables $\operatorname{dom}(i) \subseteq$ $\mathcal{X}$ to be held fixed, and the values to which they are fixed. Intervention $i$ induces a mapping of SCMs, also denoted $i$, so that $i(\mathfrak{M})$ is identical to $\mathfrak{M}$, but with $f_{X}$ replaced by the constant function $f_{X}(\cdot)=i(X)$ for each $X \in \operatorname{dom}(i)$. We say $i$ is finite whenever $\operatorname{dom}(i)$ is finite.

Using this definition we introduce a relation of direct causal influence (Halpern and Pearl 2005). We say $X \rightsquigarrow Y$ when there are endogenous settings $\mathfrak{x}_{1}, \mathfrak{x}_{2}$ differing only in their assignment to $X$, and an exogenous setting $\mathfrak{u}$ such that $f_{Y}\left(\mathfrak{x}_{1}, \mathfrak{u}\right) \neq f_{Y}\left(\mathfrak{x}_{2}, \mathfrak{u}\right)$. This in turn induces a dag $\mathcal{G}_{\mathfrak{M}}=(\mathcal{X}, \rightsquigarrow)$. Throughout we restrict attention to SCMs $\mathfrak{M}$ such that $\mathcal{G}_{\mathfrak{M}}$ is well-founded, generalizing the common acyclicity condition. This is also equivalent to the requirement for infinite SCMs from (Ibeling and Icard 2019).

We say $\mathfrak{M}$ is Markov if each variable is $P_{\mathfrak{M}}$-independent of its non-descendants (in $\mathcal{G}_{\mathfrak{M}}$ ) conditional on its parents. The Markov condition is guaranteed provided the exogenous variables $\mathcal{U}$ are jointly independent (Pearl 2009, Thm. 1.4.1). We define $d$-separation on a dag $\mathcal{G}$ standardly, and write $((\mathbf{X} \Perp \mathbf{Y}) \mid \mathbf{Z})_{\mathcal{G}}$ to say that the variables $\mathbf{X}$ are d-separated from $\mathbf{Y}$ given $\mathbf{Z}$. In Markov structures d-separation guarantees conditional independence.

In order to establish a correspondence (Thm. 12) with probabilistic programs, we introduce a further restriction on SCMs, following previous work on computable causal models (Janzing and Schölkopf 2010; Ibeling and Icard 2019). Def. 3 below assumes a very simple "coin-flip" probability space; we leave it as an exercise to show that there is no loss of generality compared to using any computable probability space as in, e.g., (Ackerman, Freer, and Roy 2019), including any standard continuous probability distributions.

Definition 3. $\mathfrak{M}=(\mathcal{F}, P)$ is computable if (1) its exogenous variables consist of infinitely many binary $U_{1}, U_{2}, \ldots$, each uniformly distributed by $P$, and (2) the collection $\mathcal{F}=$ $\left\{f_{X}\right\}_{X \in \mathcal{X}}$ is uniformly computable (Weihrauch 2000).

Call a model $\mathfrak{M}=(\mathcal{F}, P)$ measurable if under every finite intervention $i$, the joint distribution $P_{i(\mathfrak{M})}(\mathcal{X})$ is welldefined. The next Fact is straightforward.

Fact 1. A computable SCM is both Markov and measurable.

Proof. A computable $\mathfrak{M}=\left(\left\{f_{X}\right\}_{X \in \mathcal{X}}, P\right)$ is Markov simply because all exogenous variables are jointly independent. Let $\mathfrak{x}$ be an endogenous setting and let $u=\bigcap_{X \in \mathcal{X}} u_{X}$ where $u_{X}=\left\{\mathfrak{u}: f_{X}(\mathfrak{x}, \mathfrak{u})=\mathfrak{x}(X)\right\}$. Letting $\Sigma$ be our $\sigma$-algebra, $P_{i(\mathfrak{M})}(\mathfrak{x})$ is well-defined if $u \in \Sigma$. It suffices to show that $u_{X} \in \Sigma$ for all $X$ since $\Sigma$ closes under countable intersection. There is a machine that halts outputting the value $f_{X}(\mathfrak{x}, \mathfrak{u})$ for any $\mathfrak{u} \in u_{X}$. By then it has seen only finitely many exogenous bits, whose values we write in a finite vector $\vec{u}(\mathfrak{u})$. Thus the cylinder set $C(\vec{u}(\mathfrak{u}))$ of $\mathfrak{u}^{\prime}$ that agree with 
$\vec{u}(\mathfrak{u})$ wherever the latter is defined is contained in $u_{X}$. So letting $u_{X}^{\prime}=\bigcup_{\mathfrak{u} \in u_{X}} C(\vec{u}(\mathfrak{u})), u_{X}^{\prime} \subseteq u_{X}$. Every cylinder is in $\Sigma$ and there are only countably many cylinders, so $u_{X}^{\prime} \in \Sigma$; obviously $u_{X} \subseteq u_{X}^{\prime}$ so $u_{X} \in \Sigma$ as desired.

Let $\mathcal{M}$ be the class of all measurable SCMs that have a well-founded causal influence graph. Further, let $\mathcal{M}^{*}$ be the subclass of computable models in $\mathcal{M}$.

\section{Syntax}

\section{Probabilistic Conditionals}

We define a succession of language fragments as follows, where $X \in \mathcal{X}$ and $x \in \operatorname{Val}(X)$ :

$$
\begin{aligned}
\mathcal{L}_{\text {int }} & ::=\top|| X=x \mid \mathcal{L}_{\text {int }} \wedge \mathcal{L}_{\text {int }} \\
\mathcal{L}_{\text {prop }} & ::=X=x\left|\neg \mathcal{L}_{\text {prop }}\right| \mathcal{L}_{\text {prop }} \wedge \mathcal{L}_{\text {prop }} \\
\mathcal{L}_{\text {cond }} & ::=\left[\mathcal{L}_{\text {int }}\right] \mathcal{L}_{\text {prop }} \\
\mathcal{L}_{\text {full }} & ::=\mathcal{L}_{\text {cond }}\left|\neg \mathcal{L}_{\text {full }}\right| \mathcal{L}_{\text {full }} \wedge \mathcal{L}_{\text {full }}
\end{aligned}
$$

Based on these fragments we define a sequence of three increasingly expressive probabilistic languages $\left\{\mathcal{L}_{i}\right\}_{i=1,2,3}$. Each language $\mathcal{L}_{i}$ speaks about probabilities over the base language $\mathcal{L}_{i}^{\text {base }}$. The base languages are

$$
\mathcal{L}_{1}^{\text {base }}=\mathcal{L}_{\text {prop }}, \quad \mathcal{L}_{2}^{\text {base }}=\mathcal{L}_{\text {cond }}, \quad \mathcal{L}_{3}^{\text {base }}=\mathcal{L}_{\text {full }} .
$$

Our languages describe facts about the probabilities that base language formulas hold. As our formulas are finitary, such facts correspond to polynomials in these probabilities. Let us make this precise. Fixing a set $V$, define the polynomial terms in the variables $V$ to be the $\mathbf{t}$ generated by this grammar (where $V$ generates any element of $V$ ):

$$
\mathbf{t}::=V|\mathbf{t}+\mathbf{t}| \mathbf{t} \cdot \mathbf{t} \mid-\mathbf{t} .
$$

Then the terms of $\mathcal{L}_{i}$ are polynomials over probabilities of base formulas, i.e., polynomial terms in the variables $\left\{\mathbb{P}(\varphi): \varphi \in \mathcal{L}_{i}^{\text {base }}\right\}$. The language $\mathcal{L}_{i}, i=1,2,3$, is then a propositional language of term inequalities:

$$
\mathcal{L}_{i}::=\mathbf{t} \geqslant \mathbf{t} \quad\left|\neg \mathcal{L}_{i} \quad\right| \quad \mathcal{L}_{i} \wedge \mathcal{L}_{i}
$$

where $\mathbf{t}$ is a term of $\mathcal{L}_{i}$. We employ the following abbreviations. For $\mathcal{L}_{\text {prop }}$ and $\mathcal{L}_{\text {full }}$ we take $\perp$ to stand for any propositional contradiction, and $T$ for any propositional tautology. For terms: $\underline{0}$ for $\mathbb{P}(\perp), \underline{1}$ for $\mathbb{P}(\top)$. For $\mathcal{L}_{i}$ formulas, we write $\mathbf{t}_{1} \equiv \mathbf{t}_{2}$ for $\left(\mathbf{t}_{1} \geqslant \mathbf{t}_{2}\right) \wedge\left(\mathbf{t}_{2} \geqslant \mathbf{t}_{1}\right)$, and $\mathbf{t}_{1}>\mathbf{t}_{2}$ for $\left(\mathbf{t}_{1} \geqslant \mathbf{t}_{2}\right) \wedge \neg\left(\mathbf{t}_{2} \geqslant \mathbf{t}_{1}\right)$. Note that we may use any rational number as a term via representing its numerator as a sum of 1 s and clearing its denominator through an inequality $\mathbf{t} \geqslant \mathbf{t}$, and we write $\underline{q}$ for a rational $q$ thus considered as a term.

Strictly speaking, $\mathbb{P}(\beta)$ for $\beta \in \mathcal{L}_{\text {prop }}$ is not a well-formed term in $\mathcal{L}_{2}$ or $\mathcal{L}_{3}$. We will nonetheless use this notation with the understanding that $\mathbb{P}(\beta)$ is a shorthand for $\mathbb{P}([\top] \beta) . \mathcal{L}_{2}$ and $\mathcal{L}_{3}$ thus extend $\mathcal{L}_{1}$ in this sense.

$\mathcal{L}_{1}, \mathcal{L}_{2}$, and $\mathcal{L}_{3}$ correspond to the three levels of the causal hierarchy as proposed by (Shpitser and Pearl 2008; Pearl 2009); see also (Bareinboim et al. 2020). $\mathcal{L}_{1}$ is simply the language of probability, capturing statements like $\mathbb{P}(Y=y \mid X=x) \geqslant 1 / 2$, which is shorthand for expression $(\mathbb{P}(\top)+\mathbb{P}(\top)) \cdot \bar{P}(X=x \wedge Y=y) \geqslant \mathbb{P}(X=x) \cdot \mathcal{L}_{2}$ encompasses assertions about so-called causal effects, e.g., statements like $\mathbb{P}([X=x] Y=y) \geqslant \underline{q}$.

\section{Semantics}

A model is simply a measurable $\mathrm{SCM} \mathfrak{M}=(\mathcal{F}, P)$. Since $\mathfrak{M}$ is well-founded, each $\mathfrak{u}$ determines the values of all endogenous variables. Thus, for $\varphi \in \mathcal{L}_{\text {prop }}$ we will write $\mathcal{F}, \mathfrak{u}=\varphi$, defined in the obvious way. For $\alpha \in \mathcal{L}_{\text {int }}$ we define the intervention operation $i_{\alpha}$ so that $i_{\alpha}(\mathcal{F})$ is the result of applying the interventions specified by $\alpha$ to $\mathcal{F}$ (Def. 2). Then we say $\mathcal{F}, \mathfrak{u}=[\alpha] \beta$ just in case $i_{\alpha}(\mathcal{F}), \mathfrak{u} \models \beta$. We have thus defined $\mathcal{F}, \mathfrak{u} \models \varphi$ for all $\varphi \in \mathcal{L}_{\text {full }}$.

Finally, for any $\varphi \in \mathcal{L}$ and model $\mathfrak{M}$ define the set $S_{\mathfrak{M}}(\varphi)=\{\mathfrak{u}: \mathcal{F}, \mathfrak{u} \models \varphi\}$. Measurability of $\mathfrak{M}$ guarantees that $S_{\mathfrak{M}}(\varphi)$ is always measurable. Toward specifying the semantics of $\mathcal{L}_{i}$ we define $\llbracket \mathbf{t} \rrbracket_{\mathfrak{M}}$ recursively, with the crucial clause given by $\llbracket \mathbb{P}(\varphi) \rrbracket_{\mathfrak{M}}=P\left(S_{\mathfrak{M}}(\varphi)\right)$. Satisfaction of $\varphi \in \mathcal{L}_{i}$ is as expected: $\mathfrak{M} \vDash \mathbf{t}_{1} \geqslant \mathbf{t}_{2}$ iff $\llbracket \mathbf{t}_{\mathbf{1}} \rrbracket_{\mathfrak{M}} \geq \llbracket \mathbf{t}_{\mathbf{2}} \rrbracket_{\mathfrak{M}}$, $\mathfrak{M} \vDash \neg \varphi$ iff $\mathfrak{M} \not \models \varphi$, and $\mathfrak{M} \vDash \varphi \wedge \psi$ iff $\mathfrak{M} \vDash \varphi$ and $\mathfrak{M} \vDash \psi$. As in previous work, it is easy to see that none of the languages $\mathcal{L}_{1}, \mathcal{L}_{2}, \mathcal{L}_{3}$ is compact (Perović et al. 2008; Ibeling and Icard 2018; 2019); consequently Thm. 5 shows weak completeness only.

\section{Comparing Expressivity}

With a precise semantic interpretation of our three languages in hand, we can now show rigorously that they form a strict hierarchy, in the sense that models may be distinguishable only by moving up to higher levels of the hierarchy.

Proposition 1. $\mathcal{L}_{2}$ is strictly more expressive than $\mathcal{L}_{1}$.

Proof. Consider $\mathfrak{M}_{1}$ with $U \sim \operatorname{Bernoulli}(0.5)$ and $X:=U$ while $Y:=X$; in $\mathfrak{M}_{2}$ we have $Y:=U$ and $X:=$ $Y$. It is easy to see by an induction on terms in $\mathcal{L}_{1}$ that $\llbracket \mathbf{t} \rrbracket_{\mathfrak{M}_{1}}=\llbracket \mathbf{t} \rrbracket_{\mathfrak{M}_{2}}$, and thus $\mathfrak{M}_{1}$ and $\mathfrak{M}_{2}$ validate the same $\mathcal{L}_{1}$ formulas. Yet, $\mathfrak{M}_{1} \vDash \mathbb{P}([X=1] Y=1) \equiv \underline{1}$, while $\mathfrak{M}_{2} \not \models \mathbb{P}([X=1] Y=1) \equiv \underline{1}$. In particular the schema $\mathbb{P}(\beta \mid \alpha) \equiv \mathbb{P}([\alpha] \beta)$ is also falsified by $\mathfrak{M}_{2}$, a reflection of the distinction between observation and intervention.

Proposition 2. $\mathcal{L}_{3}$ is strictly more expressive than $\mathcal{L}_{2}$.

Proof. Consider an example adapted from (Avin, Shpitser, and Pearl 2005) with two endogenous and two exogenous variables $X, U_{X}, Y, U_{Y}$, where $U_{X} \sim \operatorname{Bernoulli}(0.5)$ and $X=U_{X}$, while $U_{Y} \sim \operatorname{Unif}(0,1,2)$. The difference between models $\mathfrak{M}_{1}$ and $\mathfrak{M}_{2}$ is the equation for binary variable $Y$. In $\mathfrak{M}_{1}$ we have $Y$ equal to $\left(X \leftrightarrow U_{Y}=0\right)$, and in $\mathfrak{M}_{2}$ we have $Y$ given by $\left(X \rightarrow U_{Y}=0\right) \wedge\left(U_{Y}=2 \rightarrow X\right)$. It is then easy to check (by induction) that $\mathfrak{M}_{1}$ and $\mathfrak{M}_{2}$ validate all the same $\mathcal{L}_{2}$ formulas, whereas, e.g., $\llbracket \mathbf{p} \rrbracket_{\mathfrak{M}_{1}} \neq \llbracket \mathbf{p} \rrbracket_{\mathfrak{M}_{2}}$, with $\mathbf{p}$ the term denoting the probability of necessity and sufficiency $\mathbb{P}([X=0] Y=0 \wedge[X=1] Y=1)$.

Note also that $\llbracket \mathbb{P}([\top] Y=1 \wedge[X=1] Y=1) \rrbracket_{\mathfrak{M}_{1}} \quad \neq$ $\llbracket \mathbb{P}([\top] Y=1 \wedge[X=1] Y=1) \rrbracket_{\mathfrak{M}_{2}}$ in this second example, showing that even allowing simple conjunctions of the form $\gamma \wedge[\alpha] \beta$ would increase the expressive power of $\mathcal{L}_{2}$. It is thus not possible in general to reason in $\mathcal{L}_{2}$ about conditional expressions such as $\mathbb{P}([\alpha] \beta \mid \gamma)$. On the other hand $\mathcal{L}_{2}$ does 
handle conditional effects, since, e.g., $\mathbb{P}([\alpha] \beta \mid[\alpha] \gamma) \geqslant \mathbf{t}$ can be rewritten as $\mathbb{P}([\alpha](\beta \wedge \gamma)) \geqslant \mathbf{t} \cdot \mathbb{P}([\alpha] \gamma){ }^{1}$

In a companion manuscript (Bareinboim et al. 2020) we improve upon Props. 1 and 2 by showing that for $i<j$ the $\mathcal{L}_{i}$-theory of a model almost-never determines its $\mathcal{L}_{j}$ theory, in the sense that the proportion of models where such collapse occurs goes asymptotically to zero.

\section{Graph Definability and $D o-$ Calculus}

Given the languages and interpretation considered so far, we mention as an aside that it may be enlightening to consider a notion of graph validity, analogous to "frame validity" in modal logic (van Benthem 2001). Let us say $\mathcal{G} \vDash \varphi$ just in case $\mathfrak{M} \vDash \varphi$ for all Markov structures $\mathfrak{M}$ such that $\mathcal{G}=\mathcal{G}_{\mathfrak{M}}$.

For any dag $\mathcal{G}$ there is a probability distribution $P$ whose conditional independencies are exactly those implied by dseparation in $\mathcal{G}$ (Geiger and Pearl 1990). It is then easy to construct an SCM $\mathfrak{M}$ with $\mathcal{G}=\mathcal{G}_{\mathfrak{M}}$ and $P=P_{\mathfrak{M}}$, which immediately gives: ${ }^{2}$

Proposition 3. $\mathcal{G} \vDash \mathbb{P}(\mathbf{X} \wedge \mathbf{Y} \mid \mathbf{Z}) \equiv \mathbb{P}(\mathbf{X} \mid \mathbf{Z}) \mathbb{P}(\mathbf{Y} \mid \mathbf{Z})$ if and only if $((\mathbf{X} \Perp \mathbf{Y}) \mid \mathbf{Z})_{\mathcal{G}}$. In other words, the graph property of d-separation is definable in $\mathcal{L}_{1}$.

One of the most intriguing components of structural causal reasoning is the do-calculus (Pearl 1995; 2009; Zhang 2008), allowing the derivation of causal effects from observational data. This calculus can also be seen as involving graph validity. The next proposition is a slight extension of what was already proved in (Pearl 1995), combined with the result of (Geiger and Pearl 1990) mentioned above.

Proposition 4. Let $\mathcal{G}$ be a dag over variables $\mathcal{X}{ }^{3}$ Then (1) $\mathcal{G} \vDash \mathbb{P}([\mathbf{X}] \mathbf{Y} \mid[\mathbf{X}](\mathbf{Z} \wedge \mathbf{W})) \equiv \mathbb{P}([\mathbf{X}] \mathbf{Y} \mid[\mathbf{X}] \mathbf{W})$ iff $((\mathbf{Y} \Perp \mathbf{Z}) \mid \mathbf{X}, \mathbf{W})_{\mathcal{G}_{\overline{\mathbf{X}}}} ;(2) \mathcal{G} \vDash \mathbb{P}([\mathbf{X} \wedge \mathbf{Z}] \mathbf{Y} \mid[\mathbf{X} \wedge \mathbf{Z}] \mathbf{W}) \equiv$ $\mathbb{P}([\mathbf{X}] \mathbf{Y} \mid[\mathbf{X}](\mathbf{Z} \wedge \mathbf{W}))$ iff $((\mathbf{Y} \Perp \mathbf{Z}) \mid \mathbf{X}, \mathbf{W})_{\mathcal{G}_{\overline{\mathbf{X}} \mathbf{z}}}$; and (3) $\mathcal{G} \vDash \mathbb{P}([\mathbf{X} \wedge \mathbf{Z}] \mathbf{Y} \mid[\mathbf{X} \wedge \mathbf{Z}] \mathbf{W}) \equiv \mathbb{P}([\mathbf{X}] \mathbf{Y} \mid[\mathbf{X}] \mathbf{W})$ iff $((\mathbf{Y} \Perp \mathbf{Z}) \mid \mathbf{X}, \mathbf{W})_{\mathcal{G}_{\overline{\mathbf{X}}, \overline{\mathbf{Z}(\mathbf{W})}}}$. All formulas here are in $\mathcal{L}_{2}$.

We leave further exploration of questions about graph definability in these languages for a future occasion.

\section{Axiomatizations}

We now give systems $A X_{i}$ each of which axiomatizes the validities of $\mathcal{L}_{i}$ over both $\mathcal{M}$ and $\mathcal{M}^{*}$. These probabilistic logics build on the base (deterministic) logics, as any equivalent base formulas must be assigned the same probability. We call $\varphi \in \mathcal{L}_{i}^{\text {base }}$ an $\mathcal{L}_{i}^{\text {base }}$-validity, and write $\models_{\mathcal{L}_{i}^{\text {base }}} \varphi$, if for all $\mathcal{F}$ and $\mathfrak{u}$ we have $\mathcal{F}, \mathfrak{u} \models \varphi$. For $\varphi \in \mathcal{L}_{\text {prop }}$, write $=\varphi$ if $\varphi$ is a propositional tautology. The validities of $\mathcal{L}_{3}^{\text {base }}=\mathcal{L}_{\text {full }}$

\footnotetext{
${ }^{1}$ In the notation of $d o$-calculus (Pearl 1995; 2009), the expression $\mathbb{P}([\alpha] \beta \mid[\alpha] \gamma)$ would be written as $\mathbb{P}(\beta \mid d o(\alpha), \gamma)$.

${ }^{2} \mathbb{P}(\mathbf{X} \wedge \mathbf{Y} \mid \mathbf{Z}) \equiv \mathbb{P}(\mathbf{X} \mid \mathbf{Z}) \mathbb{P}(\mathbf{Y} \mid \mathbf{Z})$ represents a conjunction over all instances of this schema with all combinations of values $\mathbf{X}=\mathbf{x}$, $\mathbf{Y}=\mathbf{y}, \mathbf{Z}=\mathbf{z}$. Here $\mathbf{X}, \mathbf{Y}, \mathbf{Z}$ are lists of variables and $\mathbf{x}, \mathbf{y}, \mathbf{z}$ are corresponding lists of values in the respective ranges.

${ }^{3} \mathcal{G} \overline{\mathbf{X} Z}$ is $\mathcal{G}$ minus any edges into $\mathbf{X}$ or out of $\mathbf{Z}$, and $\mathbf{Z}(\mathbf{W})$ is the set of all $\mathbf{Z}$-nodes that are not ancestors of any $\mathbf{W}$-node in $\mathcal{G}_{\overline{\mathbf{X}}}$.
}

have been axiomatized in (Ibeling and Icard 2019). Suppose $\varphi=[\alpha] \beta \in \mathcal{L}_{2}^{\text {base }}=\mathcal{L}_{\text {cond. }}$. It is easy to see that $\models_{\mathcal{L}_{2}^{\text {base }}} \varphi$ just in case $\models \alpha \rightarrow \beta$. It is even easier to see that for $\varphi \in \mathcal{L}_{1}^{\text {base }}=\mathcal{L}_{\text {prop }}, \models_{\mathcal{L}_{1}^{\text {base }}} \varphi$ just in case $\models \varphi$. Satisfiability for every base language is NP-complete. We need to add one more axiom schema, ${ }^{4}$ for all $X \in \mathcal{X}$ :

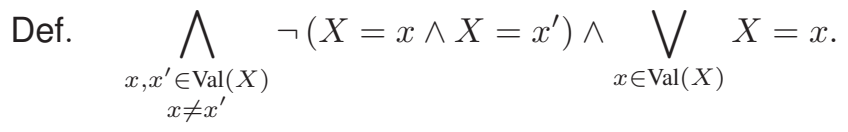

The system $\mathrm{AX}_{i}$ for $i=1,3$ is then as follows.

$\begin{aligned} \text { MP. } & \text { Inference rule: } \varphi, \varphi \rightarrow \psi \vdash \psi \\ \text { Bool. } & \text { Boolean tautologies over } \mathcal{L}_{i} \\ \text { NonNeg. } & \mathbb{P}(\varphi) \geqslant \underline{0} \\ \text { Add. } & \mathbb{P}(\varphi \wedge \psi)+\mathbb{P}(\varphi \wedge \neg \psi) \equiv \mathbb{P}(\varphi) \\ \text { Dist. } & \mathbb{P}(\varphi)=\mathbb{P}(\psi) \text { whenever } \models_{\mathcal{L}_{i}^{\text {base }}} \varphi \leftrightarrow \psi \\ \text { Poly. } & \text { The polynomial schemata below. }\end{aligned}$

The following 15 axioms constitute Poly.

$$
\begin{aligned}
\text { OrdTot. } & \mathbf{t}_{1} \geqslant \mathbf{t}_{2} \vee \mathbf{t}_{2} \geqslant \mathbf{t}_{1} \\
\text { OrdTrans. } & \mathbf{t}_{1} \geqslant \mathbf{t}_{2} \wedge \mathbf{t}_{2} \geqslant \mathbf{t}_{3} \rightarrow \mathbf{t}_{1} \geqslant \mathbf{t}_{3} \\
\text { NonDegen. } & \neg(\underline{0} \equiv \underline{1}) \\
\text { AddComm. } & \mathbf{t}_{1}+\mathbf{t}_{2} \equiv \mathbf{t}_{2}+\mathbf{t}_{1} \\
\text { AddAssoc. } & \left(\mathbf{t}_{1}+\mathbf{t}_{2}\right)+\mathbf{t}_{3} \equiv \mathbf{t}_{1}+\left(\mathbf{t}_{2}+\mathbf{t}_{3}\right) \\
\text { Zero. } & \mathbf{t}+\underline{0} \equiv \mathbf{t} \\
\text { AddOrd. } & \mathbf{t}_{1} \geqslant \mathbf{t}_{2} \rightarrow \mathbf{t}_{1}+\mathbf{t}_{3} \geqslant \mathbf{t}_{2}+\mathbf{t}_{3} \\
\text { MulComm. } & \mathbf{t}_{1} \cdot \mathbf{t}_{2} \equiv \mathbf{t}_{2} \cdot \mathbf{t}_{1} \\
\text { MulAssoc. } & \left(\mathbf{t}_{1} \cdot \mathbf{t}_{2}\right) \cdot \mathbf{t}_{3} \equiv \mathbf{t}_{1} \cdot\left(\mathbf{t}_{2} \cdot \mathbf{t}_{3}\right) \\
\text { One. } & \mathbf{t} \cdot \underline{1} \equiv \mathbf{t} \\
\text { MuIDist. } & \mathbf{t}_{1} \cdot\left(\mathbf{t}_{2}+\mathbf{t}_{3}\right) \equiv \mathbf{t}_{1} \cdot \mathbf{t}_{2}+\mathbf{t}_{1} \cdot \mathbf{t}_{3} \\
\text { MulNonNeg. } & \mathbf{t}_{1} \geqslant \underline{0} \wedge \mathbf{t}_{2} \geqslant \underline{0} \rightarrow \mathbf{t}_{1} \cdot \mathbf{t}_{2} \geqslant \underline{0} \\
\text { ZeroMul. } & \mathbf{t} \cdot \underline{0} \equiv \underline{0} \\
\text { NoZeroDiv. } & \mathbf{t}_{1} \cdot \mathbf{t}_{2} \equiv \underline{0} \rightarrow \mathbf{t}_{1} \equiv \underline{0} \vee \mathbf{t}_{2} \equiv \underline{0} \\
\text { Neg. } & \mathbf{t}+(-\mathbf{t}) \equiv \underline{0}
\end{aligned}
$$

As for $\mathrm{AX}_{2}$, Add turns out to be too weak-it only captures consequents of the trivial antecedent $(T)$ since a purely propositional $\varphi$ in the base language is interpreted as $[T] \varphi$. Thus to obtain $\mathrm{AX}_{2}$, we form the axiomatization as above, but add the following axiom Add2:

$$
\text { Add2. } \quad \mathbb{P}([\alpha](\varphi \wedge \psi))+\mathbb{P}([\alpha](\varphi \wedge \neg \psi)) \equiv \mathbb{P}([\alpha] \varphi) \text {. }
$$

\section{Sample Derivation}

Before proving completeness (Thm. 5) we illustrate the power of $\mathrm{AX}_{2}$ (and $\mathrm{AX}_{3}$ ) through a representative derivation. Our goal is to derive the example in (Pearl 1995, §3.2):

$$
\mathbb{P}\left(\left[x^{*}\right] y^{*}\right) \equiv \sum_{z} \mathbb{P}\left(z \mid x^{*}\right) \sum_{x} \mathbb{P}\left(y^{*} \mid x \wedge z\right) \mathbb{P}(x)
$$

${ }^{4}$ In (Ibeling and Icard 2019), Def merely amounts to the law of excluded middle since $\operatorname{Val}(X)=\{0,1\}$ for all $X \in \mathcal{X}$; here we assume only that every $\operatorname{Val}(X)$ is finite. 


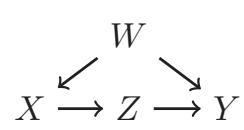

Figure 1: A graph over which (2)-(6) are all valid.

for any specific values $x^{*}$ and $y^{*}$. (Rather than writing, e.g., $X=x$, we are simply writing $x$.) This formula (in $\mathcal{L}_{2}$ ) is not in general valid. But it does follow from further assumptions easily statable in $\mathcal{L}_{2}$. Formulas (2)-(4) below are instances of the second do-calculus schema, while (5) and (6) are instances of the third schema (recall Prop. 4).

$$
\begin{aligned}
\mathbb{P}([X] Z) & \equiv \mathbb{P}(Z \mid X) \\
\mathbb{P}([X] Y \mid[X] Z) & \equiv \mathbb{P}([X \wedge Z] Y) \\
\mathbb{P}([Z] Y \mid[Z] X) & \equiv \mathbb{P}(Y \mid X \wedge Z) \\
\mathbb{P}([X \wedge Z] Y) & \equiv \mathbb{P}([Z] Y) \\
\mathbb{P}([Z] X) & \equiv \mathbb{P}(X)
\end{aligned}
$$

Prop. 4 provides the graphical assumptions needed to justify each of these assertions. For example, they are all valid over the graph in Fig. 1 (Pearl 1995; 2009). We now argue that $((2) \wedge(3) \wedge(4) \wedge(5) \wedge(6)) \rightarrow(1)$ is derivable in our calculus. ${ }^{5}$ Notably, this can be done in $\mathcal{L}_{2}$ using only $\mathrm{AX}_{2}$. First, by appeal to MP, Bool, and Dist, we have that $\mathbb{P}\left(\left[x^{*}\right] y^{*}\right) \equiv \mathbb{P}\left(\bigvee_{z}\left(\left[x^{*}\right] y^{*} \wedge\left[x^{*}\right] z\right)\right)$, which in turn using Add2 is equal to $\sum_{z} \mathbb{P}\left(\left[x^{*}\right] y^{*} \wedge\left[x^{*}\right] z\right)$. By Poly this can be shown equal to $\sum_{z}^{z} \mathbb{P}\left(\left[x^{*}\right] z\right) \mathbb{P}\left(\left[x^{*}\right] y^{*} \mid\left[x^{*}\right] z\right)$. By (2) and (3) this is equal to $\sum_{z}^{z} \mathbb{P}\left(z \mid x^{*}\right) \mathbb{P}\left(\left[x^{*} \wedge z\right] y^{*}\right)$, and by (5) to $\sum_{z} \mathbb{P}\left(z \mid x^{*}\right) \mathbb{P}\left([z] y^{*}\right)$. Employing a similar argument to that above (using MP, Bool, Add2, and Dist), this is equal to $\sum_{z} \mathbb{P}\left(z \mid x^{*}\right) \sum_{x} \mathbb{P}\left([z] y^{*} \mid[z] x\right) \mathbb{P}([z] x)$. By (4) and (6) we finally obtain $\sum_{z} \mathbb{P}\left(z \mid x^{*}\right) \sum_{x} \mathbb{P}\left(y^{*} \mid x \wedge z\right) \mathbb{P}(x)$.

\section{Completeness Theorems}

Theorem 5. Each $\mathrm{AX}_{i}$ is sound and complete for the validities of $\mathcal{L}_{i}$ with respect to both $\mathcal{M}$ and $\mathcal{M}^{*}$.

Proof. Soundness is straightforward. For completeness, we show any consistent $\varphi \in \mathcal{L}_{i}$ is satisfiable. There is a consistent clause in its disjunctive normal form so we may assume that $\varphi$ is a conjunction of literals (using MP, Bool). We can now obtain a normal form for $\varphi$ as in (Fagin, Halpern, and Megiddo 1990). Lem. 6 below gives this for the $i=1,3$ case. We will show later how to modify it (Lem. 9) for $A X_{2}$.

Lemma 6. Suppose $\varphi \in \mathcal{L}_{i}$ is a conjunction of literals. Let $a_{1}, \ldots, a_{n} \in \mathcal{L}_{i}^{\text {base }}$ be the base atoms appearing in $\varphi$. Let $\Delta=\left\{\ell_{1} \wedge \cdots \wedge \ell_{n}: \ell_{i} \in\left\{a_{i}, \neg a_{i}\right\}\right.$ for all $\left.1 \leq i \leq n\right\}$. Let $\Delta_{\perp}=\left\{\delta: \models \mathcal{L}_{i}^{\text {base }} \delta \leftrightarrow \perp\right\}$. Then there are polynomial terms

\footnotetext{
${ }^{5}$ It is worth observing that this derivation would go through even if we considered the weaker logic for $\mathcal{L}_{\text {full }}$ studied in (Ibeling and Icard 2018). That is, deriving $((2) \wedge(3) \wedge(4) \wedge(5) \wedge(6)) \rightarrow(1)$ does not depend on any of the causal axioms that characterize structural causal models (Halpern 2000; Pearl 2009). However, slightly weaker assumptions-e.g., $\mathbb{P}([X] Z) \equiv \mathbb{P}([X] Z \mid X)$ in place of (2) — would require the additional axioms.
}

$\mathbf{t}_{1}, \ldots, \mathbf{t}_{m}, \mathbf{t}_{1}^{\prime}, \ldots, \mathbf{t}_{m^{\prime}}^{\prime}$ in the variables $\{\mathbb{P}(\delta)\}_{\delta \in \Delta}$ such that $\varphi$ is provably-in- $\mathrm{AX}_{i}$ equivalent to a conjunction

$$
\begin{gathered}
\bigwedge_{\delta \in \Delta_{\perp}} \mathbb{P}(\delta) \equiv \underline{0} \wedge \bigwedge_{\delta \in \Delta \backslash \Delta_{\perp}} \mathbb{P}(\delta) \geqslant \underline{0} \wedge \sum_{\delta \in \Delta} \mathbb{P}(\delta) \equiv \underline{1} \\
\wedge \bigwedge_{1 \leq i \leq m} \mathbf{t}_{i} \geqslant \underline{0} \wedge \bigwedge_{1 \leq i \leq m^{\prime}} \mathbf{t}_{i}^{\prime}>\underline{0} .
\end{gathered}
$$

Proof. The first part of (7) comes from Dist, the second from NonNeg, and the remainder from Add and Poly. This is most clearly illustrated by example. Consider $\varphi=$ $\mathbb{P}([X] Y)+\underline{2} \mathbb{P}([Y] Z) \equiv 1 / 2$ so that $\Delta=\left\{\delta_{1}=[X] Y \wedge\right.$ $[Y] Z, \delta_{2}=[X] Y \wedge \neg[Y] Z, \delta_{3}=\neg[X] Y \wedge[Y] Z, \delta_{4}=$ $\neg[X] Y \wedge \neg[Y] Z\}$ and $\Delta_{\perp}=\varnothing$. Let $p_{i}$ abbreviate $\mathbb{P}\left(\delta_{i}\right)$. By NonNeg, $\vdash\left(p_{1} \geqslant \underline{0}\right) \wedge \cdots \wedge\left(p_{4} \geqslant \underline{0}\right)$ and by Add, $\vdash p_{1}+\cdots+p_{4} \equiv \underline{1}$. Now, we may compute the $\left\{\mathbf{t}_{i}, \mathbf{t}_{i}^{\prime}\right\}$ in (7). Using Poly and Add:

$$
\begin{aligned}
\vdash \varphi & \leftrightarrow \underline{2} \mathbb{P}([X] Y)+\underline{4} \mathbb{P}([Y] Z)-\underline{1} \equiv \underline{0} \\
\vdash \varphi & \leftrightarrow \underline{2} \mathbb{P}([X] Y \wedge[Y] Z)+\underline{2} \mathbb{P}([X] Y \wedge \neg[Y] Z) \\
+ & \underline{4} \mathbb{P}([X] Y \wedge[Y] Z)+\underline{4} \mathbb{P}(\neg[X] Y \wedge[Y] Z)-\underline{1} \equiv \underline{0} \\
\vdash \varphi & \leftrightarrow \underline{6} p_{1}+\underline{2} p_{2}+\underline{4} p_{3}-\underline{1} \equiv \underline{0} \\
\vdash \varphi & \leftrightarrow \underline{6} p_{1}+\underline{2} p_{2}+\underline{4} p_{3}-\underline{1} \leqslant \underline{0} \\
\wedge & -\underline{6} p_{1}-\underline{2} p_{2}-\underline{4} p_{3}+\underline{1} \leqslant \underline{0}
\end{aligned}
$$

so that $m=2, m^{\prime}=0$ in $(7)$, and $\mathbf{t}_{1}$ is $\underline{6} p_{1}+\underline{2} p_{2}+\underline{4} p_{3}-\underline{1}$ while $\mathbf{t}_{2}$ is $-\mathbf{t}_{1}$. We can carry out this process for any $\varphi$, with $m^{\prime} \neq 0$ if $\varphi$ contains negations.

(7) is a system of polynomial inequalities in the unknowns $\{\mathbb{P}(\delta)\}_{\delta \in \Delta}$. We now demonstrate this system has a solution provided (7) is consistent. Our primary tool is the following semialgebraic result (Stengle 1974).

Theorem 7 (Positivstellensatz). Let $R=\mathbb{Q}\left[x_{1}, \ldots, x_{n}\right]$ and suppose $f_{1}, \ldots, f_{s}, g_{1}, \ldots, g_{t}, h_{1}, \ldots, h_{m} \in R$. Let cone $\left(g_{1}, \ldots, g_{t}\right) \subseteq R$ be the closure of $\left\{g_{1}, \ldots, g_{t}\right\} \cup$ $\left\{s^{2}: s \in R\right\}$ under addition and multiplication, and let ideal $\left(h_{1}, \ldots, h_{m}\right)=\left\{\sum_{i=1}^{m} a_{i} h_{i}: a_{1}, \ldots, a_{m} \in R\right\}$. Then either the system $\left\{f_{i} \neq 0, g_{j} \geq 0, h_{k}=0: 1 \leq i \leq s, 1 \leq\right.$ $j \leq t, 1 \leq k \leq m\}$ has a solution over $\mathbb{R}^{n}$, or there exist $g \in \operatorname{cone}\left(g_{1}, \ldots, g_{t}\right), h \in \operatorname{ideal}\left(h_{1}, \ldots, h_{m}\right), n \in \mathbb{N}$ with

$$
g+h+F^{2 n}=0
$$

where $F=\prod_{1 \leq i \leq s} f_{i}$.

Each clause in (7) easily translates to a polynomial in Thm. 7; a clause $\mathbf{t}_{i}^{\prime}>\underline{0}$ becomes two constraints: $\mathbf{t}_{i}^{\prime} \geq 0$ and $\mathbf{t}_{i}^{\prime} \neq 0$. If there's no solution, let $\mathbf{t}=(\mathbf{g}+\mathbf{h})+\mathbf{f}^{2 n}$ for some $\mathbf{g}, \mathbf{h}, \mathbf{f}$ as in (8), where $\mathbf{f}^{2 n}$ is an iterated multiplication. We claim $\varphi \vdash \mathbf{t} \equiv \underline{0} \wedge \mathbf{t}>\underline{0}$ so that $\varphi$ is inconsistent, a contradiction. We use the principles below, all derivable from Poly:

$$
\begin{aligned}
\text { AddPos. } & \mathbf{t}_{1} \geqslant \underline{0} \wedge \mathbf{t}_{2}>\underline{0} \rightarrow \mathbf{t}_{1}+\mathbf{t}_{2}>\underline{0} \\
\text { MulPos. } & \mathbf{t}_{1}>\underline{0} \wedge \mathbf{t}_{2}>\underline{0} \rightarrow \mathbf{t}_{1} \cdot \mathbf{t}_{2}>\underline{0} \\
\text { NegAdd. } & -\left(\mathbf{t}_{1}+\mathbf{t}_{2}\right) \equiv\left(-\mathbf{t}_{1}\right)+\left(-\mathbf{t}_{2}\right) \\
\text { NegNeg. } & -(-\mathbf{t}) \equiv \mathbf{t} \\
\text { OrdSq. } & \mathbf{t} \cdot \mathbf{t} \geqslant \underline{0}
\end{aligned}
$$


First, we show $\varphi \vdash \mathbf{t}>\underline{0}$. Note that $\varphi \vdash \mathbf{g} \geqslant \underline{0}$ by OrdSq and $\varphi \vdash \mathbf{h} \equiv \underline{0}$ by ZeroMul given Thm. 7 and the clauses of (7). Also, $\varphi \vdash \mathbf{f}>\underline{0}$ by NonDegen if $m^{\prime}=0$ in (7) or $n=0$ in (8) and by MulPos otherwise. So $\varphi \vdash(\mathbf{g}+\mathbf{h})+\mathbf{f}^{2 n}>\underline{0}$ by AddPos. Now we show $\varphi \vdash \mathbf{t} \equiv \underline{0}$. In fact, we don't need $\varphi$. We show that Poly is powerful enough to simplify polynomials; then by soundness and since (8) holds identically, $\vdash \mathbf{t} \equiv \underline{0}$. Using MulDist, NegAdd, $\vdash \mathbf{t} \equiv \mathbf{m}$ where $\mathbf{m}$ is a sum of non- $\underline{0}$ monomials, each of which is either 1 itself or contains no factors of 1 (One), and contains at most one - sign (NegNeg). By MulComm, MulAssoc group the factors in each leftassociatively and in increasing (lexicographic) order of their variables. Then with AddComm, AddAssoc, Neg group and cancel out to $\underline{0}$ equal but opposite monomials. Adding all the $\underline{0}$ s, we have $\vdash$ m $\equiv \underline{0}$.

The $\{\mathbb{P}(\delta)\}_{\delta \in \Delta}$ can be assumed computable, as there is an algebraic (Tarski 1949) and a fortiori computable solution to (7). Now, this implies there is a $\mathfrak{M} \in \mathcal{M}^{*} \subset \mathcal{M}$ that satisfies each $\delta \in \Delta \backslash \Delta_{\perp}$ with probability $\mathbb{P}(\delta)$, so that $\mathfrak{M} \models \varphi$ :

Lemma 8. Let $\left\{\varphi_{i}\right\}_{1<i<N}$ be satisfiable $\mathcal{L}_{\text {full-formulas no }}$ pair of which is jointly satisfiable, and let $\left\{p_{i}\right\}_{1 \leq i \leq N}$ be nonnegative computable reals summing to unity. Then there is a $\mathfrak{M} \in \mathcal{M}^{*}$ such that $\llbracket \mathbb{P}\left(\varphi_{i}\right) \rrbracket_{\mathfrak{M}}=p_{i}$ for all $1 \leq i \leq N$.

Proof. (Ibeling and Icard 2019) give semantics of $\mathcal{L}_{\text {full }}$ over deterministic SCMs, i.e., those in which $\mathcal{U}=\varnothing$. Thus there are determinstic $M_{i}=\left(\left\{f_{X, i}\right\}_{X \in \mathcal{X}}, \cdot\right)$ such that $M_{i}=\varphi_{i}$ for all $i$. Consider $\mathfrak{M}=(\mathcal{F}, P)$ with one exogenous variable $\mathcal{U}=\{U\}$ such that $\operatorname{Val}(U)=\{1, \ldots, N\}$, where for all $i$ the probability that $U=i$ is $p_{i}$. Define $\mathcal{F}=\left\{f_{X}\right\}_{X \in \mathcal{X}}$ as $f_{X}(\mathfrak{x}, i)=f_{X, i}(\mathfrak{x})$ for any endogenous setting $\mathfrak{x}$. When $U=i$, the structural equations of $\mathfrak{M}$ and $M_{i}$ coincide, so $\mathcal{F}, i \models \varphi_{i}$. Conversely, if $\mathcal{F}, i \models \varphi_{j}$, then $j=i$ : otherwise $M_{i} \models \varphi_{i} \wedge \varphi_{j}$. Thus $S_{\mathfrak{M}}\left(\varphi_{i}\right)=\{i\}$ and $\llbracket \mathbb{P}\left(\varphi_{i}\right) \rrbracket \mathfrak{M}=p_{i}$ for all $i$. Clearly $\mathfrak{M}$ can be made to satisfy Def. 3 .

As for the $A X_{2}$ case, Lem. 6 must be modified, but the proof is the same (no elements of $\Delta$ are jointly satisfiable):

Lemma 9. Suppose $\varphi \in \mathcal{L}_{2}$ is a conjunction of literals. Let $a_{1}, \ldots, a_{n} \in \mathcal{L}_{\text {prop }}$ be the $\mathcal{L}_{\text {prop }}$-atoms appearing in $\varphi$ (i.e. its subformulas of the form $X=x$ ) and let $\alpha_{1}, \ldots, \alpha_{l} \in \mathcal{L}_{\text {int }}$ be the antecedents of any conditionals appearing in $\varphi$. Let $\Delta_{\text {prop }}=\left\{\ell_{1} \wedge \cdots \wedge \ell_{n}: \ell_{i} \in\left\{a_{i}, \neg a_{i}\right\}\right.$ for all $\left.1 \leq i \leq n\right\}$ and let $\Delta=\left\{\left[\alpha_{i}\right] \delta_{\text {prop }}: 1 \leq i \leq l, \delta_{\text {prop }} \in \Delta_{\text {prop }}\right\}$. Let $\Delta_{\perp}=\left\{\delta: \models_{\mathcal{L}_{2}} \delta \leftrightarrow \perp\right\}$. Then there are polynomial terms $\mathbf{t}_{1}, \ldots, \mathbf{t}_{m}, \mathbf{t}_{1}^{\prime}, \ldots, \mathbf{t}_{m^{\prime}}^{\prime}$ in the variables $\{\mathbb{P}(\delta)\}_{\delta \in \Delta}$ such that $\varphi$ is provably-in- $\mathrm{AX}_{2}$ equivalent to a conjunction

$$
\begin{gathered}
\bigwedge_{\delta \in \Delta_{\perp}} \mathbb{P}(\delta) \equiv \underline{0} \wedge \bigwedge_{\delta \in \Delta \backslash \Delta_{\perp}} \mathbb{P}(\delta) \geqslant \underline{0} \wedge \\
\bigwedge_{1 \leq i \leq l} \sum_{\delta_{\text {prop }} \in \Delta_{\text {prop }}} \mathbb{P}\left(\left[\alpha_{i}\right] \delta_{\text {prop }}\right) \equiv \underline{1} \\
\wedge \bigwedge_{1 \leq i \leq m} \mathbf{t}_{i} \leqslant \underline{0} \wedge \bigwedge_{1 \leq i \leq m^{\prime}} \mathbf{t}_{i}^{\prime}>\underline{0} .
\end{gathered}
$$

Proof. Use Add2 in place of Add in proving Lem. 6.

\section{Complexity}

Let PROB-CAUSAL-SAT $i$ be the problem of deciding if a given formula $\varphi \in \mathcal{L}_{i}$, encoded standardly, is satisfiable. The completeness proof above delivers no obvious complexity bound, but we will now obtain a polynomial space bound by extending an argument of (Fagin, Halpern, and Megiddo 1990). As PSPACE closes under complement, this also shows the validity problem for our languages is in PSPACE. The crux is a small-model property:

Lemma 10. Any satisfiable $\varphi$ has a small model $\mathfrak{M}$ in the sense that $\left|\left\{\delta \in \Delta: \llbracket \delta \rrbracket_{\mathfrak{M}}>0\right\}\right| \leq|\varphi|$ where $\Delta$ is defined as in Lem. 6, 9.

Proof. Let $\Psi$ be the $\mathcal{L}_{i}^{\text {base }}$-formulas appearing inside $\varphi$; we have $|\Psi|<|\varphi|$. For $\psi \in \Psi$ let $\Delta_{\psi}=\left\{\delta \in \Delta: \models_{\mathcal{L}_{i}^{\text {base }}} \delta \rightarrow\right.$ $\psi$ \}; the proof of Lem. 6 or 9 show that $\sum_{\delta \in \Delta_{\psi}} \mathbb{P}(\delta)=\mathbb{P}(\psi)$ is valid. Now let $\mathfrak{M}^{\prime}$ be such that $\mathfrak{M}^{\prime}=\varphi$ and consider the system $S$ in the unknowns $\{\mathbb{P}(\delta)\}_{\delta \in \Delta}: S=\left\{\sum_{\delta \in \Delta} \mathbb{P}(\delta)=\right.$ 1\} $\cup\left\{\sum_{\delta \in \Delta_{\psi}} \mathbb{P}(\delta)=\llbracket \psi \rrbracket_{\mathfrak{M}^{\prime}}\right\}_{\psi \in \Psi}$. By a fact of linear algebra (Fagin, Halpern, and Megiddo 1990, Lem. 2.5), since $S$ has $|\Psi|+1$ equations, it has a nonnegative solution $s$ where at most $|\Psi|+1$ variables are nonzero. Apply Lem. 8 to $s$, giving a small $\mathfrak{M}$ such that $\llbracket \psi \rrbracket_{\mathfrak{M}}=\llbracket \psi \rrbracket_{\mathfrak{M}^{\prime}}$ for all $\psi \in \Psi$, so that $\mathfrak{M} \models \varphi$.

\section{Theorem 11. Each PROB-CAUSAL-SAT $i \in$ PSPACE.}

Proof. Algorithm: for each subset $\Delta^{\prime} \subseteq \Delta$ of size $\left|\Delta^{\prime}\right| \leq$ $|\varphi|$, form a formula $\varphi_{\Delta^{\prime}}$ in the existential theory of the reals $(\exists \mathbb{R})$, over variables $\left\{p_{\delta}\right\}_{\delta \in \Delta^{\prime}}$ as follows. Conjoin the equations $\sum_{\delta \in \Delta^{\prime}} p_{\delta}=1$ and $\bigwedge_{\delta \in \Delta^{\prime}} p_{\delta} \geq 0$ to the result of replacing any $\mathbb{P}(\psi)$ appearing in $\varphi$ with $\sum_{\delta \in \Delta_{\varphi}} p_{\delta}$. Then check satisfiability of $\varphi_{\Delta^{\prime}}$ via the PSPACE decision procedure for $\exists \mathbb{R}$ of (Canny 1988). Declare $\varphi$ sat if any $\varphi_{\Delta^{\prime}}$ is satisfiable, and unsat if not. Why does it work? If $\varphi$ is satisfiable, we have a model with a small $\Delta^{\prime}$ by Lem. 10, and the corresponding $\left\{p_{\delta}\right\}_{\delta \in \Delta^{\prime}}$ are a witness for $\varphi_{\Delta^{\prime}}$. Conversely, if $\varphi_{\Delta^{\prime}}$ is satisfiable in $\exists \mathbb{R}$ for some $\Delta^{\prime}$, then apply Lem. 8 to the reals $\left\{p_{\delta}\right\}_{\delta \in \Delta^{\prime}}$, giving a model satisfying $\varphi$.

\section{Probabilistic Programs}

Thm. 5 establishes soundness and completeness for both the class of measurable SCMs, and also for the more restricted class of computable SCMs. The latter result is especially useful for establishing a link to an alternative perspective on causal modeling, emphasizing a procedural rather than declarative aspect (Icard 2017). We take a probabilistic program to be any generative algorithm:

Definition 4 (Probabilistic simulation model). A probabilistic simulation is a probabilistic Turing machine with a readonly random bit tape, a work tape, and a write-only variable tape encoding the variables $\mathcal{X}$.

A probabilistic simulation outputs values for endogenous variables, eventually establishing a complete endogenous setting $\mathfrak{x}$ on the variable tape. Thus, like a SCM, a probabilistic simulation model $\mathrm{T}$ gives a probability distribution $P_{\mathrm{T}}(\mathcal{X})$. The following definition of intervention endows these models with a genuine causal interpretation: 
Definition 5. Given a computable intervention $i$ (as in Def. 2 ) and a corresponding oracle for $i$, the simulation $i(\mathrm{~T}) \mathrm{em}-$ ulates $\mathrm{T}$ but acts as if the square for any $X \in i$ is fixed to the value $i(X)$; it dovetails this emulation with a procedure that writes $i(X)$ to $X$ for all $X \in \operatorname{dom}(i)$.

In (Ibeling and Icard 2019) a subclass $\mathcal{T}^{*}$ of simulation programs is studied, namely those (1) that satisfy a strong "functionality" property for interventions, (2) that produce a solution under every intervention, and (3) for which the direct causal influence relation is well-founded (analogously to the requirement above for SCMs). We can then obtain:

Theorem 12. For every $\mathfrak{M} \in \mathcal{M}^{*}$ there is a $T \in \mathcal{T}^{*}$ such that, for every computable intervention $i$, we have $P_{i(\mathfrak{M})}(\mathcal{X})=P_{i(\mathrm{~T})}(\mathcal{X})$, and vice versa.

Proof. The only difference between the present setting and the one in (Ibeling and Icard 2019) is the presence of an infinite sequence of random bits (recall Def. 3 for SCMs). Thus, the same construction as in (Ibeling and Icard 2019, Thm. 1) gives a model for which, when a random bit string is fixed, we have equivalence under any interventions of the endogenous variables.

With the obvious interpretation of $\mathcal{L}_{i}$ formulas over simulation models in $\mathcal{T}^{*}$, we thus have:

Corollary 13. Each $\mathrm{AX}_{i}$ is sound and complete for the validities of $\mathcal{L}_{i}$ with respect to $\mathcal{T}^{*}$.

\section{Conclusion}

We have introduced a series of increasingly expressive languages encoding levels of the "ladder of causation" (Shpitser and Pearl 2008; Pearl 2009), interpreted over both standard structural causal models and probabilistic simulation programs. We moreover established some fundamental theoretical results about these languages, including finitary axiomatizations and PSPACE complexity upper bounds for satisfiability and validity. This marks the first systematic study of a probabilistic logic of causal counterfactuals.

Along the way we also noted how logical languages might help to illuminate aspects of probabilistic causal reasoning. As a final illustration, let us return again to the do-calculus. We noted two seminal formula schemas from $\mathcal{L}_{2}$ that feature centrally in the do-calculus:

$$
\begin{gathered}
\mathbb{P}([\mathbf{X} \wedge \mathbf{Z}] \mathbf{Y} \mid[\mathbf{X} \wedge \mathbf{Z}] \mathbf{W}) \equiv \mathbb{P}([\mathbf{X}] \mathbf{Y} \mid[\mathbf{X}](\mathbf{Z} \wedge \mathbf{W})) \\
\mathbb{P}([\mathbf{X} \wedge \mathbf{Z}] \mathbf{Y} \mid[\mathbf{X} \wedge \mathbf{Z}] \mathbf{W}) \equiv \mathbb{P}([\mathbf{X}] \mathbf{Y} \mid[\mathbf{X}] \mathbf{W})
\end{gathered}
$$

Where $\Gamma$ is a set of $\mathcal{L}_{3}$ formulas, let $\Gamma_{d o}$ be all instances of (10) and (11) that can be inferred from $\Gamma$ using the rules and axioms of $\mathrm{AX}_{3}$ (equiv. are entailed by $\Gamma$ ). As usual, we say $\Gamma \vDash \varphi$ to mean that $\Gamma$ semantically entails $\varphi$, while $\Gamma \vdash \varphi$ means there is a proof in $A_{3}$ of $\varphi$ from assumptions in $\Gamma$. The completeness results of (Huang and Valtorta 2006; Shpitser and Pearl 2008), together with Thm. 5, establish the following combined completeness result:

Corollary 14. Let $\mathbf{p}$ be a term of $\mathcal{L}_{1}$ and $\chi \in \mathcal{L}_{2}^{\text {base }}=\mathcal{L}_{\text {cond }}$. Then $\Gamma \vDash \mathbb{P}(\chi) \equiv \mathbf{p}$ implies $\Gamma_{d o} \vdash \mathbb{P}(\chi) \equiv \mathbf{p}$.
In other words, to know whether a causal effect $\mathbb{P}(\chi)$ can be reduced to a pure probabilistic expression $\mathbf{p}$, given some set of assumptions $\Gamma$, it suffices to take as premises only instances of (10) and (11) (which by Prop. 4 can all be inferred from specific graphical properties), and apply the calculus $\mathrm{AX}_{3}$ (or simply $A X_{2}$ ). The derivation of (1) from (2)(6) above is a concrete illustration of this Corollary.

More generally, we submit that the formalization of causal languages offered in this paper helps to clarify what exactly the levels of the causal hierarchy come to, and how we might gain a better understanding of how they relate to each other and to tasks that an intelligent agent might need to solve. For a start on such exploration, see (Bareinboim et al. 2020).

\section{Future Work}

Our work opens up a number of possibilities for further investigation. We mention several here.

Although the complexity of decision problems for the languages considered here is relatively low compared to many expressive logical systems, it may be desirable to consider yet smaller fragments of probability logic. For example, the language of probability statements with linear inequalities remains in NP (Fagin, Halpern, and Megiddo 1990). While this fragment is too impoverished to express general assertions about conditional probability, one could extend the language only minimally; cf. (Ivanovska and Giese 2010).

It is also natural to consider more expressive languages. For instance, (Ibeling and Icard 2019) included a (deterministic) causal influence relation $\rightsquigarrow$ explicitly in the logical language, showing, e.g., that transitivity of this relation characterizes exactly the "local" SCMs (Pearl 2009). Would this characterization extend to the probabilistic interpretation of causal influence? Indeed, from the perspective of causal learning and reasoning it would be natural to include explicit statements about the underlying graph in the logical syntax (Geiger and Pearl 1990; Hyttinen, Eberhardt, and Järvisalo 2014; 2015; Triantafillou and Tsamardinos 2015).

On the other hand, leaving graph properties merely implicit as we have done here raises numerous theoretical questions about graph definability, as briefly explored above. Analogous to the case of modal logic, we can ask for the class of graphical properties that can be defined by $\mathcal{L}_{1}, \mathcal{L}_{2}$, or $\mathcal{L}_{3}$. Considering different types of causal graphs may lead to variations of this question, e.g., with mixed ancestral graphs (Spirtes, Glymour, and Scheines 2000) which we know reveal a different version of Prop. 4 (Zhang 2008).

Finally, while Props. 1 and 2 report known results establishing basic strictness of the hierarchy, it would of course be desirable to develop a much more comprehensive and systematic theory of expressiveness for the three languages, again akin to what we have for many other logical languages. What kinds of invariance properties do these languages imply? We leave these open questions for future work.

\section{Acknowledgments}

This material is based upon work supported by the National Science Foundation Graduate Research Fellowship Program under Grant No. DGE-1656518, and by the Center for the Study of Language and Information. 


\section{References}

Ackerman, N. L.; Freer, C. E.; and Roy, D. M. 2019. On the computability of conditional probability. Journal of the ACM 66(3).

Avin, C.; Shpitser, I.; and Pearl, J. 2005. Identifiability of path-specific effects. In Proceedings of the International Joint Conference on Artificial Intelligence (IJCAI).

Bareinboim, E.; Correa, J.; Ibeling, D.; and Icard, T. 2020. Foundations of causal inference and the emergence of Pearl's hierarchy. manuscript.

van Benthem, J. 2001. Correspondence theory. In Gabbay, D. M., and Guenthner, F., eds., Handbook of Philosophical Logic, volume 3. Springer.

Bingham, E.; Chen, J. P.; Jankowiak, M.; Obermeter, F.; Pradhan, N.; Karaletsos, T.; Singh, R.; Szerlip, P.; Horsfall, P.; and Goodman, N. D. 2019. Pyro: Deep universal probabilistic programming. Journal of Machine Learning Research 28:1-6.

Canny, J. 1988. Some algebraic and geometric computations in PSPACE. In Proceedings of the Twentieth Annual ACM Symposium on Theory of Computing, STOC '88, 460-467. New York, NY, USA: ACM.

Fagin, R.; Halpern, J. Y.; and Megiddo, N. 1990. A logic for reasoning about probabilities. Information and Computation $87: 78-128$.

Galles, D., and Pearl, J. 1998. An axiomatic characterization of causal counterfactuals. Foundations of Science 3(1):151182.

Geiger, D., and Meek, C. 1999. Quantifier elimination for statistical problems. In Proceedings of the Fifteenth Conference on Uncertainty in Artificial Intelligence, UAI'99, 226235. San Francisco, CA, USA: Morgan Kaufmann Publishers Inc.

Geiger, D., and Pearl, J. 1990. On the logic of causal models. Machine Intelligence and Pattern Recognition 9:3-14.

Halpern, J. Y., and Pearl, J. 2005. Causes and Explanations: A Structural-Model Approach. Part I: Causes. The British Journal for the Philosophy of Science 56(4):843-887.

Halpern, J. Y. 2000. Axiomatizing causal reasoning. Journal of Artificial Intelligence Research 12:317-337.

Huang, Y., and Valtorta, M. 2006. Pearl's calculus of intervention is complete. In Proceedings of the 22nd Conference on Uncertainty in Artificial Intelligence (UAI).

Hyttinen, A.; Eberhardt, F.; and Järvisalo, M. 2014. Constraint-based causal discovery: Conflict resolution with answer set programming. In Proceedings of the Thirtieth Conference on Uncertainty in Artificial Intelligence (UAI).

Hyttinen, A.; Eberhardt, F.; and Järvisalo, M. 2015. Docalculus when the true graph is unknown. In Proceedings of the Thirty-First Conference on Uncertainty in Artificial Intelligence (UAI).

Ibeling, D., and Icard, T. 2018. On the conditional logic of simulation models. In Proceedings of the 27th International Joint Conference on Artificial Intelligence (IJCAI 2018).
Ibeling, D., and Icard, T. 2019. On open-universe causal reasoning. In Proceedings of the Conference on Uncertainty in Artificial Intelligence (UAI).

Ibeling, D. 2018. Causal modeling with probabilistic simulation models. In Proceedings of the 5th International Workshop on Probabilistic Logic Programming (PLP), 36-48.

Icard, T. F. 2017. From programs to causal models. In Cremers, A.; van Gessel, T.; and Roelofsen, F., eds., Proceedings of the 21st Amsterdam Colloquium, 35-44.

Ivanovska, M., and Giese, M. 2010. Probabilistic logics with conditional independence formulae. In 19th European Conference on Artificial Intelligence (ECAI).

Janzing, D., and Schölkopf, B. 2010. Causal inference using the algorithmic Markov condition. IEEE Transactions on Information Theory 56(10):5168-5194.

Lake, B. M.; Ullman, T. D.; Tenenbaum, J. B.; and Gershman, S. J. 2017. Building machines that learn and think like people. Behavioral and Brain Sciences 40.

Pearl, J., and Bareinboim, E. 2012. External validity: From do-calculus to transportability across populations. Statistical Science 29(4):579-595.

Pearl, J. 1995. Causal diagrams for empirical research. Biometrika 82(4):669-710.

Pearl, J. 2009. Causality. CUP.

Perović, A.; Ognjanović, Z.; Rašković, M.; and Marković, Z. 2008. A probabilistic logic with polynomial weight formulas. In International Symposium on Foundations of Information and Knowledge Systems, 239-252.

Shpitser, I., and Pearl, J. 2008. Complete identification methods for the causal hierarchy. Journal of Machine Learning Research 9:1941-1979.

Spirtes, P.; Glymour, C.; and Scheines, R. 2000. Causation, Prediction, and Search. MIT Press.

Stengle, G. 1974. A Nullstellensatz and a Positivstellensatz in semialgebraic geometry. Mathematische Annalen 207(2):87-97.

Tarski, A. 1949. A decision method for elementary algebra and geometry. Journal of Symbolic Logic 14(3):188-188.

Tavares, Z.; Koppel, J.; Zhang, X.; and Solar-Lezama, A. 2019. A language for counterfactual generative models. www.zenna.org/publications/causal.pdf.

Triantafillou, S., and Tsamardinos, I. 2015. Constraintbased causal discovery from multiple interventions over overlapping variable sets. Journal of Machine Learning Research 16:2147-2205.

Weihrauch, K. 2000. Computable Analysis. Springer Verlag. Zhang, J. 2008. Causal reasoning with ancestral graphs. Journal of Machine Learning Research 9:1437-1474.

Zhang, J. 2013. A Lewisian logic of causal counterfactuals. Minds and Machines 23:77-93. 